\title{
Polarization of MTIP is a signature of gliding locomotion in Plasmodium ookinetes and sporozoites
}

\author{
Inga Siden-Kiamos ${ }^{\mathrm{a}}$, Christian Goosmann ${ }^{\mathrm{b}}$, Carlos A. Buscaglia ${ }^{\mathrm{c}}$, Volker Brinkmann ${ }^{\mathrm{b}}$, \\ Kai Matuschewski ${ }^{\mathrm{b}, \mathrm{d}}$, Georgina N. Montagna ${ }^{\mathrm{b}, \mathrm{c}, \mathrm{e}, *}$ \\ ${ }^{\text {a } I n s t i t u t e ~ o f ~ M o l e c u l a r ~ B i o l o g y ~ a n d ~ B i o t e c h n o l o g y, ~ F o u n d a t i o n ~ f o r ~ R e s e a r c h ~ a n d ~ T e c h n o l o g y, ~ H e l l a s, ~} 70013$ Heraklion, Crete, Greece \\ ${ }^{\mathrm{b}}$ Max Planck Institute for Infection Biology, 10117 Berlin, Germany \\ ' Instituto de Investigaciones Biotecnológicas 'Dr Rodolfo Ugalde' (IIBio), UNSAM-CONICET, 1650 San Martín, Buenos Aires, Argentina \\ ${ }^{\mathrm{d}}$ Department of Molecular Parasitology, Institute of Biology, Humboldt University, 10117 Berlin, Germany \\ ${ }^{\mathrm{e}}$ Departamento de Microbiologia, Imunologia e Parasitologia, Universidade Federal de São Paulo, 049032, SP, Brazil.
}

A R T I C L E I N F O

\section{Keywords:}

Plasmodium

MTIP

Hsp20

Motility

Cell polarity

\begin{abstract}
A B S T R A C T
Gliding motility and cell invasion are essential for the successful transmission of Plasmodium parasites. These processes rely on an acto-myosin motor located underneath the parasite plasma membrane. The Myosin A-tail interacting protein (MTIP) connects the class XIV myosin A (MyoA) to the gliding-associated proteins and is essential for assembly of the motor at the inner membrane complex. Here, we assessed the subcellular localization of MTIP in Plasmodium berghei motile stages from wild-type parasites and mutants that lack MyoA or the small heat shock protein 20 (HSP20). We demonstrate that MTIP is recruited to the apical end of motile ookinetes independently of the presence of MyoA. We also show that infective sporozoites displayed a polarized MTIP distribution during gliding, and that this distribution was abrogated in mutant parasites with an aberrant locomotion.
\end{abstract}

Apicomplexan parasites have complex life cycles consisting of extensive intracellular replication, sexual recombination, and brief, extracellular motile stages that permit parasite dispersal and host switch. For instance, in order to successfully colonize the mammalian host, Plasmodium sporozoites need to migrate, traverse several tissues, and eventually invade the final host cell, a hepatocyte [1]. The proteins which constitute the molecular motor that propels the extracellular motile stages, are highly conserved across the phylum and are shared between the diverse motile and invasive stages, e.g. Plasmodium ookinetes, sporozoites and merozoites, and Toxoplasma tachyzoites [2]. The motor complex is located between the parasite plasma membrane (PM) and a unique structure of flattened Golgi-derived cisternae, termed inner membrane complex (IMC). Previous work established that the motion force is generated by a small class XIV myosin, MyoA, which in turn is indirectly anchored to the IMC. The power strokes along short filamentous actin, which is positioned underneath the PM and anchored to adhesion receptors, generate parasite locomotion along the substrate or host cell surfaces [1].

A central protein in the motor complex is the MyoA-anchoring protein, Myosin-tail interacting protein (MTIP). This protein was initially identified using the yeast two-hybrid system and by in vitro protein-binding assays [3]. The interaction domain to MTIP is confined to the carboxy-terminal 15 amino acids of MyoA [3]. This high-affinity interaction occurs independent of calcium binding, and MyoA-anchoring by MTIP likely permits the fast velocity which is comparable to muscle myosins [4]. The crystal structure of the heterodimeric MyoAMTIP complex uncovered major conformational changes in the carboxy-terminal region of MTIP upon binding to MyoA [5]. Although the structural basis for MyoA-MTIP complex has been revealed, its assembly and cellular distribution in motile parasites remain largely unknown.

Here, we analyzed the distribution of MTIP in different stages of Plasmodium berghei. For this reason, we generated a specific antiserum against MTIP. Initially, we analyzed the localization of MTIP in late schizonts of $P$. berghei. MTIP is present in the periphery of individual merozoites in mature schizonts, where it co-localizes with actin I (Fig. 1A). This staining is in good agreement with previous findings showing that MTIP co-localizes with components of the IMC [6].

In order to analyze the MTIP localization in motile stages of Plasmodium, we first studied ookinetes. For ookinete cultures, gametocyte-positive blood from mice infected with $P$. berghei ANKA was seeded and cultured for $24 \mathrm{~h}$. We observed that in motile ookinetes MTIP

\footnotetext{
* Corresponding author at: Instituto de Investigaciones Biotecnológicas 'Dr Rodolfo Ugalde' (IIBio), UNSAM-CONICET, 1650 San Martín, Buenos Aires, Argentina

E-mail address: gmontagna@iib.unsam.edu.ar (G.N. Montagna).
} 
A
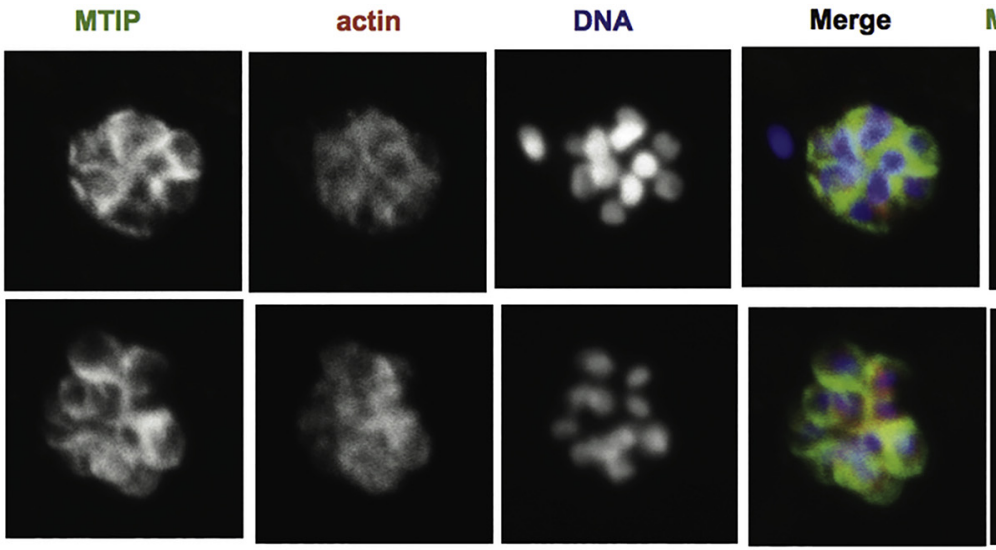

MTIPIDNA

B
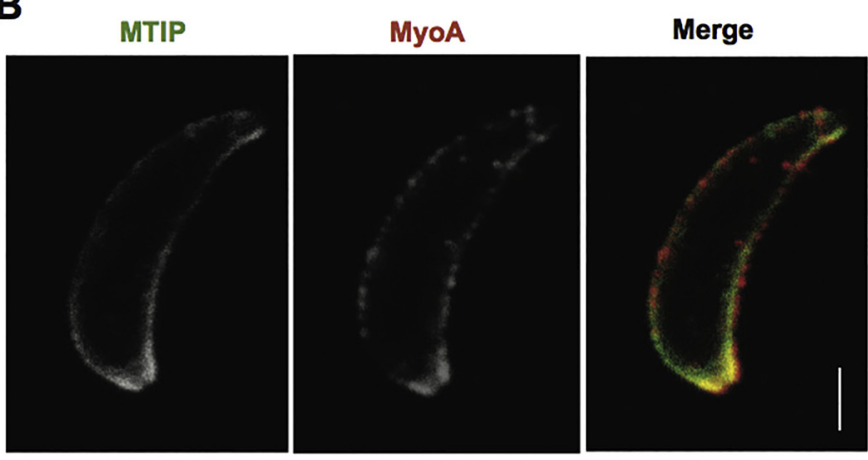

D

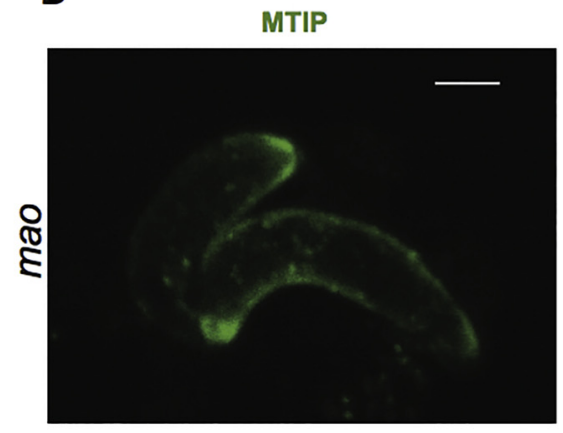

C

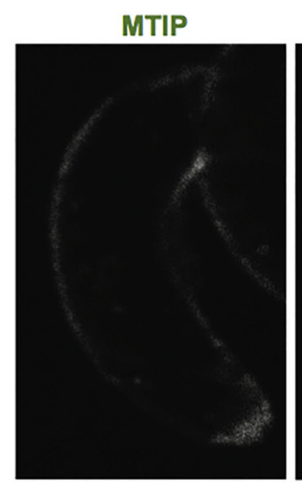

GAP50
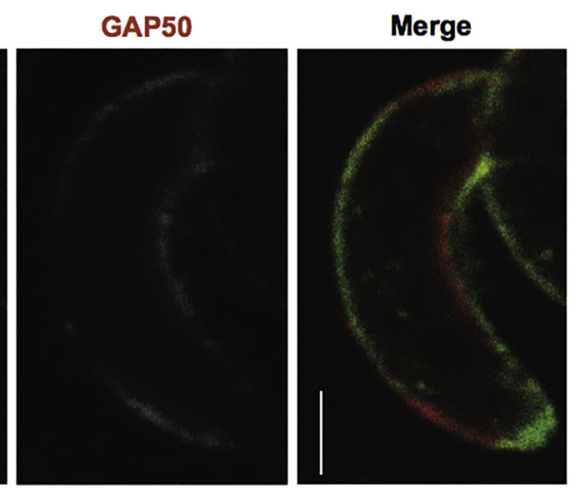

E

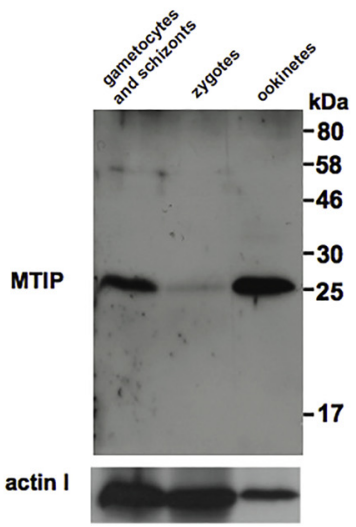

Fig. 1. MTIP polarization in motile Plasmodium stages. (A) MTIP is detected in P. berghei mature schizonts. Indirect immunofluorescence of cells fixed with $4 \%$ paraformaldehyde (PFA) in PBS and permeabilized with $0.1 \%$ Triton X-100 in PBS. Samples were incubated with an anti-P. berghei actin antibody [17] (red) and a mouse anti-MTIP antiserum (green) raised against $P$. berghei MTIP obtained after injection of the plasmid pSecTag2a-MTIP, encoding the entire open reading frame of MTIP, into BALB/C mice [18]. DNA was stained with TO-PRO (Invitrogen) (blue). Scale bar: $2 \mu \mathrm{m}$ (B) MTIP and Myo A are located at the apical tip of ookinetes. Indirect immunofluorescence assays of $P$. berghei ookinetes using mouse anti-MTIP antibody (green) and rabbit anti-MyoA antibody (red). Scale bar $2 \mu \mathrm{m}$. (C) Colocalization of GAP50 (red) and MTIP (green) in P. berghei ookinetes. Note that GAP50 signal is absent at the tip of the parasite. (D) MTIP is localized at the tip of ookinete independently of MyoA. Indirect immunofluorescence assays of $P$. berghei mao ookinetes. (E) Expression of MTIP in different stages of $P$. berghei. Western blots were performed as described previously [11]. As a loading control the blot was incubated with the antibody against actin I [17].

accumulates at the apical tip of the parasite and co-localized with MyoA (Fig. 1B). In contrast, GAP50 is distributed along the periphery of the whole ookinete body, with the notable exception of the tip, where MTIP is localized (Fig. 1C). This finding is in line with previous data, showing that the myosin light chain 1 (MLC1), the MTIP orthologue in Toxoplasma tachyzoites anchors MyoA to the glideosome-associated protein 45 (GAP45), which is integral to the IMC [7].

To investigate the localization of MTIP in MyoA-depleted P. berghei ookinetes we used a promoter-swap mutant parasite, called mao, in which myoA gene expression is turned off in ookinetes [8]. We observed that MTIP was localized at the tip of mao ookinetes (Fig. 1D) and, hence, localization was independent of the presence of MyoA protein, consistent with previous observations in T. gondii [7]. MTIP expression was also analyzed by Western blot (Fig. 1E). We observed that this protein is readily detectable in mixed gametocytes and schizonts. Remarkably, zygotes collected $3 \mathrm{~h}$ after seeding an ookinete culture showed a severe reduction in MTIP expression, whereas ookinetes exhibited the highest amount of MTIP protein (Fig. 1E). These results indicate that MTIP expression is tightly controlled during mammalian host-to-mosquito vector switch.

The polarized localization in ookinetes prompted us to revise the MTIP localization in sporozoites, the infective forms of malaria 


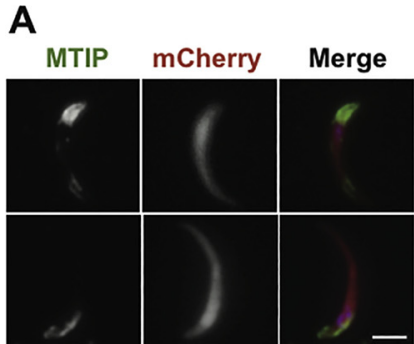

B
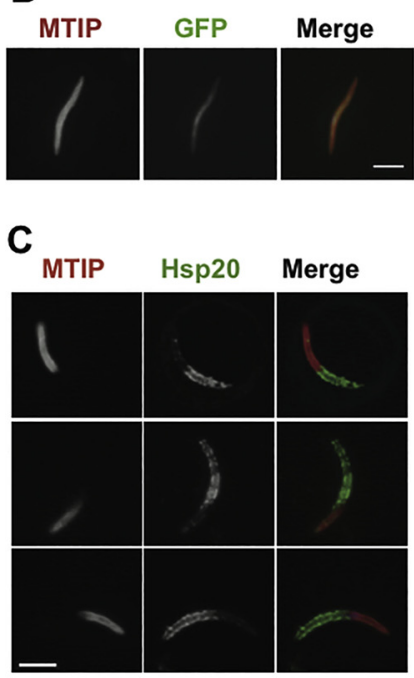

D
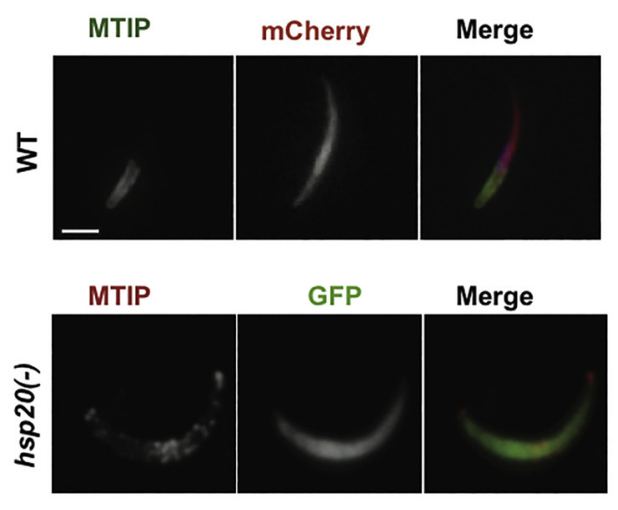

WT $9 \%$

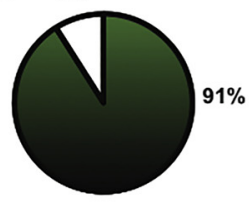

hsp20(-)

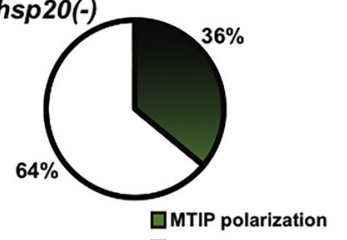

\section{E}

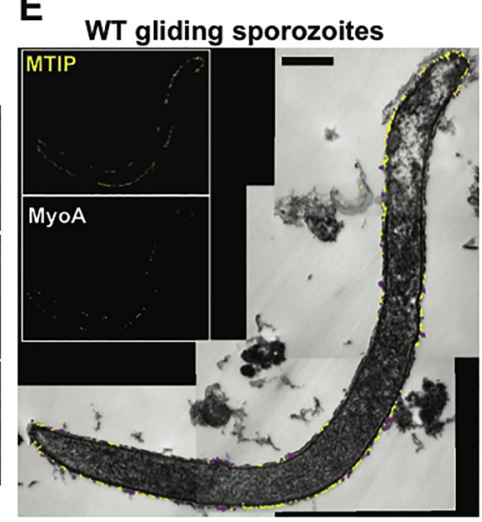

hsp20(-) gliding sporozoites

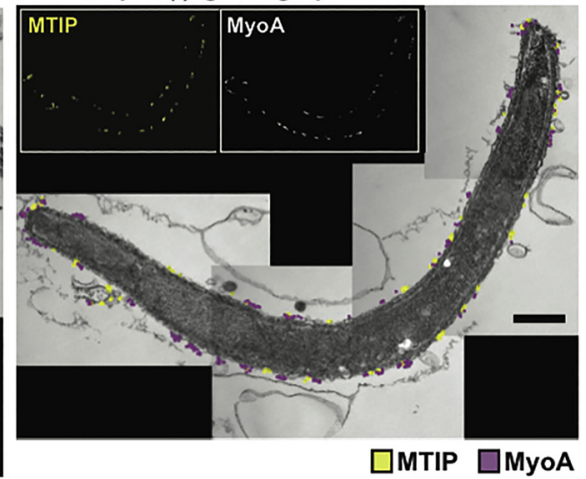

Fig. 2. Loss of MTIP polarization in hsp20(-) sporozoites.

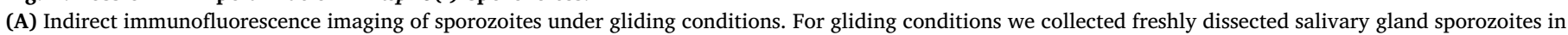

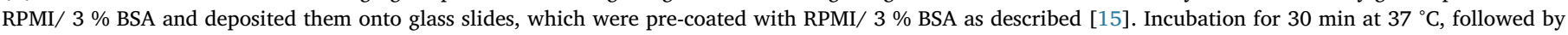

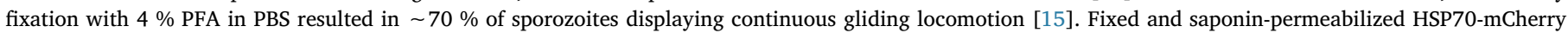

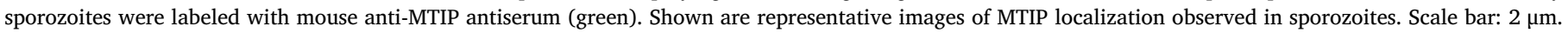

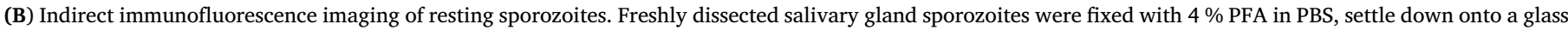

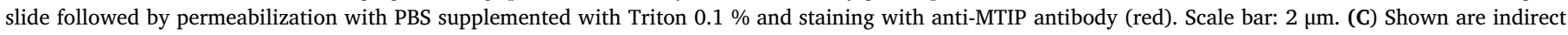

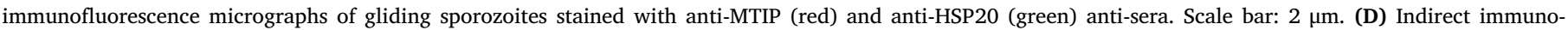

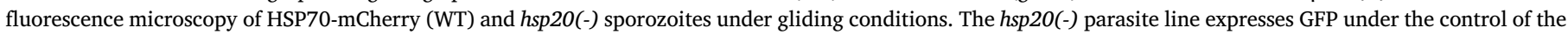

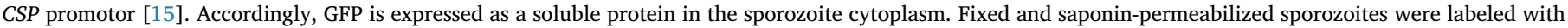

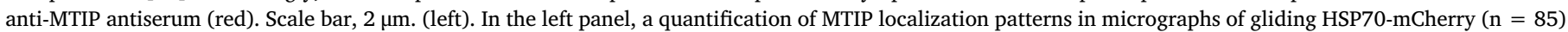

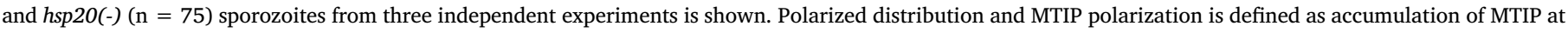

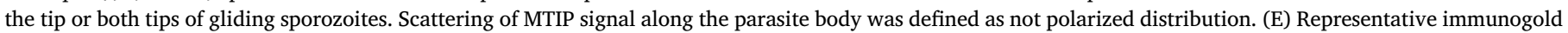

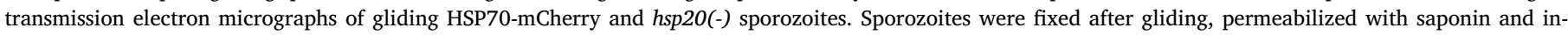

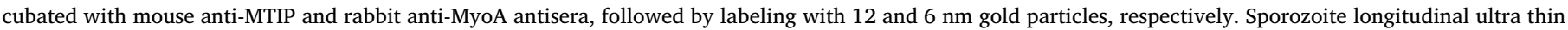

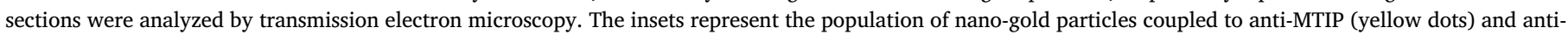

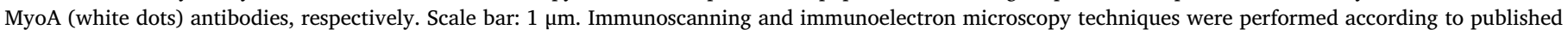
protocols [15].

parasites. Sporozoite fast gliding motility is characterized by a high turnover of small and transient adhesion sites [9]. It has been postulated that immobilized MyoA generates the force driving parasite movement, but actin dynamics also appear to regulate parasite adhesion to substrates before and during gliding [9,10]. Previous results demonstrated that several Plasmodium proteins, for instance TRAP and MyoA, are polarized and/or re-localized during gliding locomotion [11-14]. Moreover, cytoplasmic factors, e.g. HSP20, are re-localized and exposed to the sporozoite surface $[14,15]$. We have previously shown that HSP20, a small chaperone that regulates parasite motility, is polarized at the apical tip of gliding sporozoites [15]. To study if this is also the case for MTIP, we performed immunofluorescence assays and analyzed MTIP distribution in gliding transgenic sporozoites that express an HSP70-mCherry fusion protein [16]. Sporozoites were allowed to glide on a glass slide and then fixed and permeabilized with saponin. We observed that MTIP is located at the tip of gliding sporozoites
(Fig. 2A). In contrast to this observation, previous studies described a strong peripheral staining of $P$. yoelii sporozoites using a different antibody against MTIP [3]. We decided to repeat the assays in the same conditions used in the previous report. After isolation from the salivary glands, non-gliding sporozoites were fixed, permeabilized with $0.1 \%$ Triton X-100, and then stained with the MTIP antibody (Fig. 2B). As expected, we observed that under these conditions, i.e. in resting sporozoites, MTIP is distributed along the periphery of and not polarized as in gliding sporozoites [3].

To investigate if MTIP co-localizes with HSP20 in gliding sporozoites we performed immunofluorescence assays using wild-type $P$. berghei ANKA sporozoites. After fixation and saponin-permeabilization, samples were stained with mouse anti-MTIP and rabbit anti-PbHSP20 antibodies. We observed that the signals for these two proteins were mutually excluded and localized to the opposite ends of the parasites (Fig. 2C). 
We also examined the distribution of MTIP in parasites lacking HSP2O. Hsp20(-) sporozoites showed abnormally large adhesion sites that correlate with a reduced speed and aberrant trajectories leading to a substantial delay in sporozoite transmission [15]. The aberrant motility phenotype displayed by $h s p 20(-)$ parasites led us to hypothesize that MTIP might have a distorted distribution as compared to wild-type (WT) sporozoites. Therefore, we performed IFA assays during gliding locomotion (Fig. 2D). We observed that in $90 \%$ of WT sporozoites MTIP was localized at one end, while this pattern was reduced to only $36 \%$ in hsp20(-) sporozoites (Fig. 2D). The majority of mutant sporozoites showed a uniform distribution of MTIP along the entire parasite body. To analyze this differential pattern in more detail, we carried out immunogold transmission electron microscopy of gliding WT and hsp2O(-) sporozoites (Fig. 2E). We observed an accumulation of MTIP signal at the tip, and to a lesser extent, at the curvatures of gliding WT sporozoites, while this molecule followed a patchy distribution along the entire periphery in gliding $h s p 20(-)$ sporozoites. Together, these observations indicate that HSP20 might be necessary to maintain Plasmodium cell polarity, which is particularly important in mature ookinetes and sporozoites during the processes of gliding locomotion and cell invasion.

In conclusion, we observed a polarization of MTIP localization in Plasmodium ookinetes and sporozoites during gliding. Both HSP20 and MTIP were found at the parasite periphery but the signals for these two proteins were mutually excluded. Strikingly, loss of HSP2O resulted in mislocalization of MTIP during gliding, in good correlation with the aberrant motility observed in hsp20(-) parasites [15]. While MTIP/ MLC1 localization is dependent on its interaction with glideosome proteins [7], it is still unknown how HSP20 is recruited to the IMC. An attractive hypothesis is that variable spatial location of HSP20 is linked to the state of actin treadmilling or a related dynamic molecular mechanism leading to cell polarity during gliding locomotion.

\section{Declaration of Competing Interest}

The authors declare that they have no conflicts of interest with the contents of this article.

\section{Acknowledgements}

This work was funded in part by FAPESP (grants 13/14361-5 and 14/07138-0) and in part by the Max-Planck Society and the EviMalaR network funded by the European Commission. The authors declare that they have no conflicts of interest with the contents of this article.

\section{References}

[1] G.N. Montagna, K. Matuschewski, C.A. Buscaglia, Plasmodium sporozoite motility: an update, Front. Biosci. 17 (2012) 726-744.

[2] J. Baum, D. Richard, J. Healer, M. Rug, Z. Krnajski, T.W. Gilberger, J.L. Green, A.A. Holder, A.F. Cowman, A conserved molecular motor drives cell invasion and gliding motility across malaria life cycle stages and other apicomplexan parasites, J. Biol. Chem. 281 (2006) 5197-5208.

[3] L.W. Bergman, K. Kaiser, H. Fujioka, I. Coppens, T.M. Daly, S. Fox, K. Matuschewski, V. Nussenzweig, S.H. Kappe, Myosin A tail domain interacting protein (MTIP) localizes to the inner membrane complex of Plasmodium sporozoites, J. Cell Sci. 116 (2003) 39-49.

[4] J.L. Green, S.R. Martin, J. Fielden, A. Ksagoni, M. Grainger, B.Y. Yim Lim, J.E. Molloy, A.A. Holder, The MTIP-myosin A complex in blood stage malaria parasites, J. Mol. Biol. 355 (2006) 933-941.

[5] J. Bosch, S. Turley, T.M. Daly, S.M. Bogh, M.L. Villasmil, C. Roach, N. Zhou, J.M. Morrisey, A.B. Vaidya, L.W. Bergman, W.G. Hol, Structure of the MTIP-MyoA complex, a key component of the malaria parasite invasion motor, Proc. Natl. Acad. Sci. U. S. A. 103 (2006) 4852-4857.

[6] J.L. Green, R.J. Wall, J. Vahokoski, N.A. Yusuf, M.A.M. Ridzuan, R.R. Stanway, J. Stock, E. Knuepfer, D. Brady, S.R. Martin, S.A. Howell, I.P. Pires, R.W. Moon, J.E. Molloy, I. Kursula, R. Tewari, A.A. Holder, Compositional and expression analyses of the glideosome during the Plasmodium life cycle reveal an additional myosin light chain required for maximum motility, J. Biol. Chem. 292 (2017) 17857-17875.

[7] K. Frenal, J.B. Marq, D. Jacot, V. Polonais, D. Soldati-Favre, Plasticity between MyoC- and MyoA-glideosomes: an example of functional compensation in Toxoplasma gondiiinvasion, PLoS Pathog. 10 (2014) e1004504.

[8] I. Siden-Kiamos, M. Ganter, A. Kunze, M. Hliscs, M. Steinbuchel, J. Mendoza, R.E. Sinden, C. Louis, K. Matuschewski, Stage-specific depletion of myosin A supports an essential role in motility of malarial ookinetes, Cell. Microbiol. 13 (2011) 1996-2006.

[9] S. Munter, B. Sabass, C. Selhuber-Unkel, M. Kudryashev, S. Hegge, U. Engel, J.P. Spatz, K. Matuschewski, U.S. Schwarz, F. Frischknecht, Plasmodiumsporozoite motility is modulated by the turnover of discrete adhesion sites, Cell Host Microbe 6 (2009) 551-562.

[10] S. Hegge, S. Munter, M. Steinbüchel, K. Heiss, U. Engel, K. Matuschewski, F. Frischknecht, Multistep adhesion of Plasmodium sporozoites, FASEB J. 24 (2010) 2222-2234.

[11] I. Siden-Kiamos, J.C. Pinder, C. Louis, Involvement of actin and myosins in Plasmodium berghei ookinete motility, Mol. Biochem. Parasitol. 150 (2006) 308-317.

[12] H. Gao, Z. Yang, X. Wang, P. Qian, R. Hong, X. Chen, X.Z. Su, H. Cui, J. Yuan, ISP1anchored polarization of GCbeta/CDC50A complex initiates malaria ookinete gliding motility, Curr. Biol. 28 (2018) 2763-2776.

[13] K.S. Bane, S. Lepper, J. Kehrer, J.M. Sattler, M. Singer, M. Reinig, D. Klug, K. Heiss, J. Baum, A.K. Mueller, F. Frischknecht, The actin filament-binding protein coronin regulates motility in Plasmodium sporozoites, PLoS Pathog. 12 (2016) e1005710.

[14] K.E. Swearingen, S.E. Lindner, L. Shi, M.J. Shears, A. Harupa, C.S. Hopp, A.M. Vaughan, T.A. Springer, R.L. Moritz, S.H. Kappe, P. Sinnis, Interrogating the Plasmodium sporozoite surface: identification of surface-exposed proteins and demonstration of glycosylation on CSP and TRAP by mass spectrometry-based proteomics, PLoS Pathog. 12 (2016) e1005606.

[15] G.N. Montagna, C.A. Buscaglia, S. Munter, C. Goosmann, F. Frischknecht V. Brinkmann, K. Matuschewski, Critical role for heat shock protein 20 (HSP20) in migration of malarial sporozoites, J. Biol. Chem. 287 (2012) 2410-2422.

[16] M. Hliscs, C. Nahar, F. Frischknecht, K. Matuschewski, Expression profiling of Plasmodium berghei HSP70 genes for generation of bright red fluorescent parasites, PLoS One 8 (2013) e72771.

[17] I. Siden-Kiamos, C. Louis, K. Matuschewski, Evidence for filamentous actin in ookinetes of a malarial parasite, Mol. Biochem. Parasitol. 181 (2012) 186-189.

[18] K. Koussis, E. Goulielmaki, A. Chalari, C. Withers-Martinez, I. Siden-Kiamos, K. Matuschewski, T.G. Loukeris, Targeted deletion of a Plasmodium site-2 protease impairs life cycle progression in the mammalian host, PLoS One 12 (2017) e0170260. 